Bryn Mawr College

Scholarship, Research, and Creative Work at Bryn Mawr

College

1972

\title{
Nuclear-Spin Relaxation in Low-Density Molecular Hydrogen at Room Temperature
}

Peter A. Beckmann

Bryn Mawr College, pbeckman@brynmawr.edu

E. Elliott Burnell

Krovvidi Lalita

Robin L. Armstrong

Kenneth E. Kisman

See next page for additional authors

Let us know how access to this document benefits you.

Follow this and additional works at: http://repository.brynmawr.edu/physics_pubs

Part of the Physics Commons

\section{Custom Citation}

Peter A. Beckmann et al., Phys. Rev. A 6, 1684 (1972).

This paper is posted at Scholarship, Research, and Creative Work at Bryn Mawr College. http://repository.brynmawr.edu/physics_pubs/47

For more information, please contact repository@brynmawr.edu. 
Authors

Peter A. Beckmann, E. Elliott Burnell, Krovvidi Lalita, Robin L. Armstrong, Kenneth E. Kisman, and F. R. McCourt 


\title{
COMMENTS AND ADDENDA
}

The Comments and Addenda section is for short communications which are not of such urgency as to justify publication in Physical Review Litlers and are not appropriate for regular Articles. It includes only the following types of communications: (1) comments on papers previously published in The I'hysical Review or P'hysical Review Letters; (2) addenda to papers previously published in The Physical Review or l'hysical Review Letters, in which the additional information can be presented without the need for writing a complete article. Manuscripts intended for this section may be accompanied by a brief abstract for information-retrieval purposes. Accepted manuscripts will follow the same publication schedule as articles in this journal, and galleys will be sent to authors.

\section{Nuclear-Spin Relaxation in Low-Density Molecular Hydrogen at Room Temperature*}

\author{
Peter A. Beckmann, ${ }^{\dagger}$ E. Elliott Burnell, ${ }^{\dagger}$ and Krovvidi Lalita ${ }^{\S}$ \\ Department of Physics, University of British Columbia, British Columbia, Canada \\ and \\ Robin L. Armstrong and Kenneth E. Kisman" \\ Department of Physics, University of Toronto, Toronto, Canada \\ and \\ F.R. McCourt \\ Department of Chemistry, University of Waterloo, Waterloo, Canada \\ (Received 14 June 1972)
}

All theoretical approaches to the problem of nuclear-spin relaxation in molecular hydrogen aitempt to obtain expressions for the macroscopic longitudinal and transverse relaxation times $T_{1}$ and $T_{2}$ from microscopic equations governing the relaxation processes. Recently McCourt and Moraal ${ }^{1}$ have presented such a derivation based on the Waldmann-Snider kinetic equation. The conversion of the microscopic kinetic equation to a set of macroscopic equations is achieved through the formation of three moment equations. ${ }^{2}$ For a single-level system it is then a straightforward matter to complete the solution of the moment equations to obtain the Bloch equation. Molecular hydrogen at $77 \mathrm{~K}$ provides an example of such a system since essentially all of the ortho molecules are in the $j=1$ rotational state. A comparison of the experimental measurements of $\mathrm{Hardy}^{3}$ for $n-\mathrm{H}_{2}$ at $77 \mathrm{~K}$ shows that theory and experiment are in good agreement for $T_{2}$ and in reasonable agreement for $T_{1}$.

For a multilevel system, however, before it is possible to complete the solution of the moment equations to obtain the Bloch equation it is necessary to give careful consideration to the role played by energetically inelastic collisions. Several different cases can be distinguished.

Case $I$. If there are no energetically inelastic collisions then each $j$ state acts independently in the relaxation process and separate relaxation rates may be associated with each rotational level. The measured relaxation rates will then be obtained as a Boltzmann average of the relaxation rates describing the individual levels. In particular,

$$
\begin{aligned}
T_{1}^{-1}= & \sum_{j} p_{j}\left(T_{1}^{-1}\right)_{j} \\
= & \frac{2}{3} c^{2}\left\langle\frac{j(j+1) \tau_{j}^{\prime}}{\left.1+\omega^{2} \tau_{j}^{\prime 2}\right\rangle_{0}+\frac{2}{75}(2 I+3)(2 I-1)}\right. \\
& \times d^{2} a_{I}^{2}\left\langle\frac { j ( j + 1 ) } { ( 2 j - 1 ) ( 2 j + 3 ) } \tau _ { j } ^ { \prime \prime } \left(\frac{1}{1+\omega^{2} \tau_{j}^{\prime \prime 2}}\right.\right. \\
& \left.\left.+\frac{4}{1+4 \omega^{2} \tau_{j}^{\prime \prime 2}}\right)\right\rangle_{0},
\end{aligned}
$$

where $c$ and $d$ are the spin-rotation and diplar coupling constants, $I=1$ is the total nuclear spin of an ortho-hydrogen molecule, $a_{I=1}=\frac{1}{2},{ }^{4} \omega=\omega_{I}-\omega_{J}$ with $\omega_{I}$ and $\omega_{J}$ the nuclear and rotational Larmor frequencies, and $\tau_{j}^{\prime}$ and $\tau_{j}^{\prime \prime}$ are the correlation times for level $j$ associated with the spin rotation and dipolar interactions. This corresponds to the procedure given by Freed, ${ }^{5}$ Bloom et al. , ${ }^{6}$ and Chen and Snider. ${ }^{4}$

Case II. If the number of energetically inelastic collisions is large enough so that on the time scale 


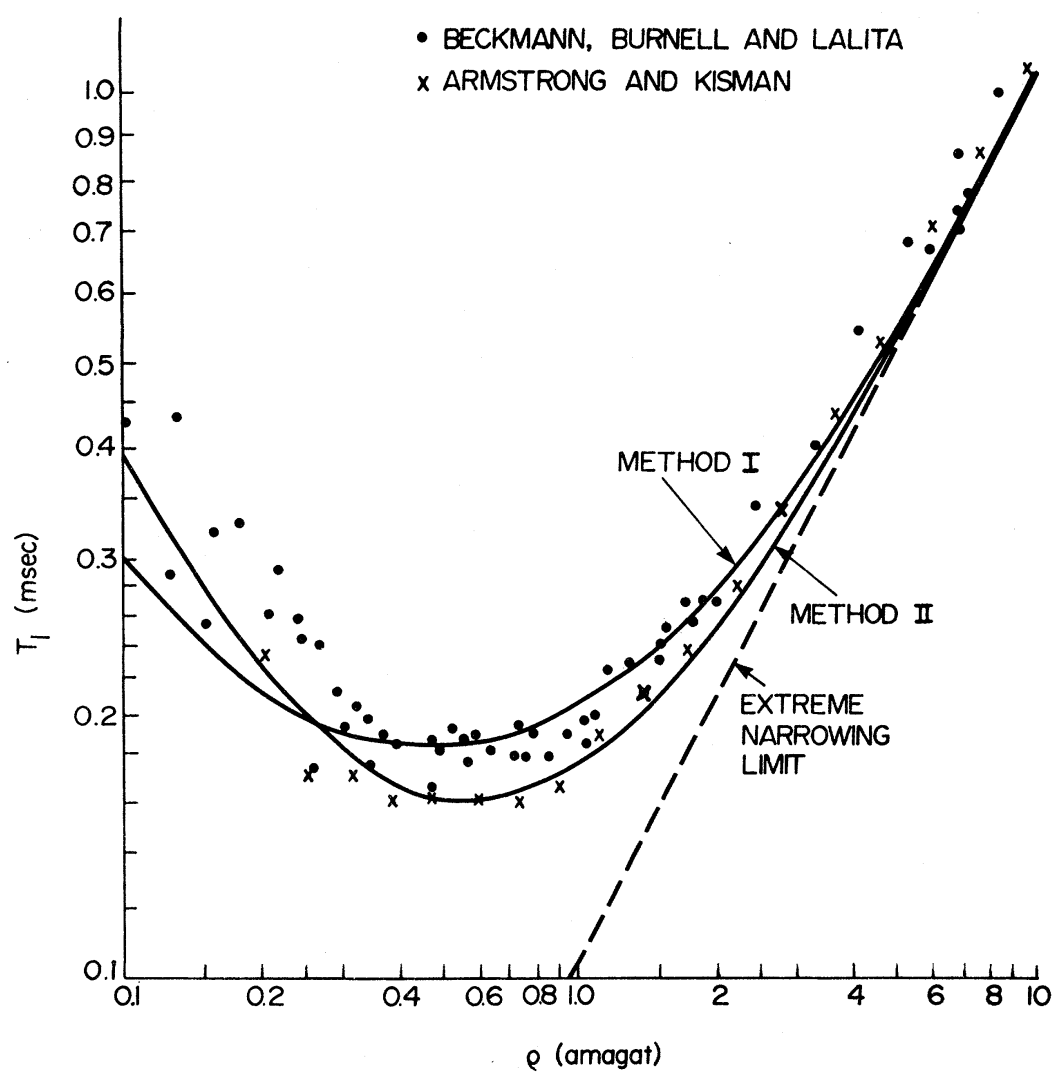

FIG. 1. Density dependence of $T_{1}$ as measured in $n-\mathrm{H}_{2}$ at $298 \mathrm{~K}$. The solid curves are theoretical calculations, for a resonance frequency of $30 \mathrm{MHz}$, based on two different methods of averaging over the rotational states. The points indicated by the closed circles were obtained at $30 \mathrm{MHz}$ and those indicated by the $\times$ 's at $28 \mathrm{MHz}$.

of the nuclear-spin relaxation experiment an ortho molecule may not be associated with a unique $j$ state, but still small enough so that they make no significant contribution to the collision integrals, then correlation times which are Boltzmann averages of the correlation times associated with specific rotational states should be taken. That is,

$$
\begin{aligned}
\left(\tau_{\mathrm{av}}^{\prime}\right)^{-1} & =\sum_{j} p_{j}\left(\tau_{j}^{\prime}\right)^{-1}=\sum_{j} p_{j}[j(j+1)]^{-1}\left(\omega_{\mathrm{col1}}^{\prime}\right)^{j}, \\
\left(\tau_{\mathrm{av}}^{\prime \prime}\right)^{-1} & =\sum_{j} p_{j}\left(\tau_{j}^{\prime \prime}\right)^{-1} \\
& =\sum_{j} p_{j}[j(j+1)(2 j-1)(2 j+3)]^{-1}\left(\omega_{\mathrm{col1}}^{\prime \prime}\right)^{j},
\end{aligned}
$$

where $\left(\omega_{\text {col1 }}^{\prime}\right)^{j}$ and $\left(\omega_{\text {col1 }}^{\prime \prime}\right)^{j}$ are the collision frequencies for state $j$ associated with the spin-rotation and dipolar interactions as deduced neglecting inelastic collisions. In this case,

$$
\begin{aligned}
T_{1}^{-1} & =\frac{2}{3} c^{2}\langle j(j+1)\rangle_{0} \frac{\tau_{\mathrm{av}}^{\prime}}{1+\omega^{2}\left(\tau_{\mathrm{av}}^{\prime}\right)^{2}}+\frac{2}{75}(2 I+3)(2 I-1) d^{2} a_{I}^{2} \\
& \times\left\langle\frac{j(j+1)}{(2 j-1)(2 j+3)}\right\rangle_{0} \tau_{\mathrm{av}}^{\prime \prime}\left(\frac{1}{1+\omega^{2}\left(\tau_{\mathrm{av}}^{\prime \prime}\right)^{2}}+\frac{4}{1+4 \omega^{2}\left(\tau_{\mathrm{av}}^{\prime \prime}\right)^{2}}\right) .
\end{aligned}
$$

Case III. If energetically inelastic collisions occur at least as frequently as elastic collisions then the $j$-dependent moment equations should be averaged over the $j$ distribution at once to give moment equations for the bulk magnetizations in the gas. That is, the $j$ states are sufficiently strongly coupled by collisions that the average correlation times are given by

$$
\begin{aligned}
\left(\tau_{\mathrm{av}}^{\prime}\right)^{-1} & =\frac{\left\langle\left(\omega_{\mathrm{col1}}^{\prime}\right)_{j}\right\rangle_{0}}{\langle j(j+1)\rangle_{0}}, \\
\left(\tau_{\mathrm{av}}^{\prime \prime}\right)^{-1} & =\frac{\left\langle\left(\omega_{\mathrm{col1}}^{\prime \prime}\right)_{j}\right\rangle_{0}}{\langle j(j+1)(2 j-1)(2 j+3)\rangle_{0}} .
\end{aligned}
$$

The relaxation rate is determined by the substitution of these expressions into (2). This corresponds to the procedure used by McCourt and Moraal. ${ }^{1}$

For molecular hydrogen at room temperature it should be sufficient to include in addition to the $j=1$ level, the $j=3$ and $j=5$ rotational levels. It is known from acoustical measurements ${ }^{7,8}$ that of the order of 1 in 300 collisions are energetically inelastic. This result implies that the contributions to collision cross sections from inelastic collisions will be less than $1 \%$. However, at 1 atm pressure, of the order of $10^{7}$ collisions occur each second which mix the rotational states. Therefore, on the time scale of a nuclear-spin relaxation experiment $\left(\sim 10^{-4} \mathrm{sec}\right)$, of the order of $10^{3}$ inelastic collisions occur. The obvious conclusion is that the averaging procedure corresponding to case (II) is to be expected experimentally. 
Measurements of $T_{1}$ through the region of the $T_{1}$ minimum in $n-\mathrm{H}_{2}$ at $298 \mathrm{~K}$ at 30 and $28 \mathrm{MHz}$ have been carried out at the University of British Columbia and the University of Toronto, respectively. The experimental techniques employed have been discussed elsewhere. ${ }^{9,10}$ The data obtained are shown in Fig. 1. Those points indicated by the symbol $\times$ represent averages of at least three data points and have an associated statistical error of $\sim \pm 5 \%$. The points indicated by the closed circle correspond to individual determinations. In the vicinity of the minimum the data represented by the $X$ 's are significantly lower than the data indicated by the closed circles. This difference reflects in part the effect of the use of different frequencies to obtain the two sets of data. Theoretically, it is predicted that the minimum value of $T_{1}$ for a resonance frequency of $28 \mathrm{MHz}$ should be $\sim 7 \%$ shorter than for a resonance frequency of $30 \mathrm{MHz}$. The residual difference can be attributed to systematic errors.
Theoretical curves were calculated using each of the three procedures outlined above using as the sole adjustable parameter the value $T_{1} / \rho=0.105$ msec amagat ${ }^{-1}$ in the extreme narrowing limit. The curves for cases I and II, at a resonance frequency of $30 \mathrm{MHz}$, are shown on the graph. The curve for case III lies between those for cases I and II but nearer to curve II. A perusal of the data in its entirety permits us to conclude that theory and experiment are in satisfactory agreement, but that the experiments have not been sufficiently refined to permit an unambiguous choice between the various methods of averaging. A better test of the theory must await more accurate experiments, preferably carried out at a higher temperature at which the problem of the choice of an averaging procedure becomes still more important.

The authors wish to thank Professor Myer Bloom for his interest in the work and T. E. Raidy for his assistance in the calculation of the theoretical curves.
* Partial support for this research was provided by the National Research Council of Canada.

†Supported by a National Research Council of Canada Bursary Grant.

* National Research Council of Canada Post Doctoral Fellow. Present address: Department of Chemistry, University of British Columbia, Canada.

§CIDA-NRCC Research Associate. Present address: Department of Physics, Indian Institute of Technology, Kanpur, U. P. , India.

"Holder of a National Research Council of Canada 1967 Science Scholarship.

${ }^{1}$ F. R. Mc Court and H. Moraal, Phys. Rev. A $\underline{5}, 2000$ (1972).

\footnotetext{
${ }^{2}$ F. R. McCourt and S. Hess, Z. Naturforsch. 25a, 1169 (1970).

${ }^{3}$ W. Hardy, Can. J. Phys. 44, 265 (1966).

${ }^{4}$ F. M. Chen and R. F. Snider, J. Chem. Phys. 48 , 3185 (1968).

${ }^{5}$ J. H. Freed, J. Chem. Phys. 41, 7 (1964).

${ }^{6}$ M. Bloom, I. Oppenheim, M. Lipsicas, C. G. Wade, and C. F. Yarnell, J. Chem. Phys. 43, 1036 (1965).

${ }^{7}$ I. F. Zartman, J. Acoust. Soc. Am. 21, 171 (1949).

${ }^{8}$ R. Brout, J. Chem. Phys. 22, 934 (1954)。

${ }^{9}$ P. A. Bechmann, M. Bloom, and E. E. Burnell, Can. J. Phys。 50, 251 (1972).

${ }^{10} \mathrm{~J}$. A。 Courtney and R. L. Armstrong, Can. J. Phys. $\underline{50}, 1252$ (1972).
}

PHYSICAL REVIEW A

VOLUME 6 , NUMBER 4

OC TOBER 1972

\title{
Convergence of Activity Expansions for Lattice Gases*
}

\author{
J. B. Hubbard \\ Department of Chemistry, Yale University, New Haven, Connecticut 06520 \\ (Received 16 February 1972)
}

\begin{abstract}
A recent theorem by Ruelle is shown to be applicable to the calculation of lower bounds on the radius of convergence of activity expansions for lattice gases. Systems with nearestneighbor repulsive interactions are used as examples. Special consideration is given to systems whose molecules possess infinitely repulsive potentials.
\end{abstract}

Systems composed of interacting molecules have played a central role in the development of statistical mechanics in spite of the fact that mathematically rigorous results for such systems are rather scarce. The compromise between resemblance to physical reality and mathematical necessity has led to the formulation of many models, some of the most successful being those that relate to lattice statistics.

Shortly after Onsager ${ }^{1}$ obtained the partition function for the square ferromagnetic Ising model, Lee and Yang ${ }^{2}$ proved that for this model and its higherdimensional analogs all the zeros of the partition function $P(z)$ reside on the unit circle in the complex 